\title{
ELECTRO-THERMAL ACTUATORS USING OPTIMIZED COMPLIANT MICROTRANSMISSIONS AS RECTILINEAR MOTION AMPLIFIERS
}

\author{
Larry L. Chu*, Joel A. Hetrick**, and Yogesh B. Gianchandani*1 \\ *Department of Electrical and Computer Engineering \\ **Department of Mechanical Engineering \\ University of Wisconsin, Madison
}

\begin{abstract}
This paper reports on the use of microtransmissions to tailor the force-displacement relationship of rectilinear electro-thermal actuators. A two-stage design approach is described for the microtransmissions, which effectively automates the synthesis procedure. It produces detailed designs of high performance compliant microtransmissions given magnification the requirements while considering buckling and minimum width (fabrication) limitations. Measurements of mechanisms fabricated from electroplated $\mathrm{Ni}$ on Si substrates demonstrate displacement amplification factors of 3-14, and match theoretical predictions within $10 \%$. Higher amplification factors can be achieved by changing certain dimensional parameters.
\end{abstract}

\section{INTRODUCTION}

Electrothermal actuators are attractive for applications that benefit from high output forces, low actuation voltages, and electrically conductive structural materials. Simple and cascaded bent-beam electrothermal actuators have been used for rectilinear motion parallel to the substrate plane $[1,2]$, producing maximum displacements and maximum blocking forces in the range of $8 \mu \mathrm{m}$ and $2.5 \mathrm{mN}$, respectively, for a silicon device of $1000 \mu \mathrm{m}$ length, $6 \mu \mathrm{m}$ width, $4.5 \mu \mathrm{m}$ thickness, and $0.2 \mathrm{rad}$. bending angle, operating at $400^{\circ} \mathrm{C}$. However, in many applications a higher displacement is necessary, a smaller force is adequate, and the operating speed of incremental mechanisms like inchworms is insufficient. In such cases, the force-displacement compromises can be tailored with compliant mechanisms that serve as backlash-free microtransmissions.

Compliant mechanisms (often called flexures) are structures that exploit elastic deformation to effectively transmit forces and motions. The advantages of compliant mechanisms include the elimination of friction, wear and backlash common in conventional mechanical joints. Due to their monolithic construction, compliant mechanisms are also much easier to fabricate at the micro-scale making them very attractive for MEMS applications.

As shown in Fig. 1, zero-backlash compliant mechanisms are useful as micro-transmissions, effectively altering the force-displacement characteristics of unconventional actuation sources such as smart materials and electro-thermal actuation sources [3]. In this paper, compliant mechanisms are used to provide efficient rectilinear motion while amplifying the strains from electrothermal bent-beam actuators. The challenge in designing a high performance compliant mechanism is to optimize the structural topology, size and geometry to efficiently transform the forces and displacements of an inpul actuation source to drive an output load.

While synthesis of compliant mechanisms [4-6] and combined electro-thermal-compliant actuators $[7,8]$ has been studied by past researchers, the approach developed here can precisely control the force-displacement trade-off of the actuator at levels of extreme magnification (up to 20X displacement amplification depending on fabrication limitations)

\section{SYNTHESIS OF COMPLIANT TRANSMISSIONS}

The design procedure is broken into topology synthesis and dimensional synthesis. Each stage is posed as a structural optimization problem, where the objective is to maximize the blocking force and unloaded output displacement given all relevant design constraints. Structural analysis is performed using structural (truss and beam) elements and linear, static finite element theory. For both topology synthesis and dimensional synthesis, the finite element equilibrium analysis and the constrained optimization problem are solved within MATLAB ${ }^{\text {TM }}$. Optimization is performed using MATLAB's Sequential Quadratic Programming (SQP) algorithm. Design derivatives are calculated using the adjoint variable method and by directly differentiating the stiffness matrix with respect to size and node position design variables.

The magnification factor, $M$, is expressed as a function of the slope change between the input and output force-displacement characteristics:

$$
M=\sqrt{\frac{d_{\max 2 \cdot F_{\text {block } 1}}}{d_{\max 1} \cdot F_{\text {block } 2}}} .
$$

where $d_{\max }$ denotes displacement in the absence of external loading forces.

While slope change is one method for determining the magnification factor, other methods such as specifying the ratio of unloaded output displacement or blocking force can also be readily accommodated. The generic structural optimization problem (for both topology and dimensional synthesis) is posed as follows:

$$
\begin{aligned}
& \text { Maximize } \quad F_{\text {block } 2} \cdot d_{\max 2}-P\left(\frac{d_{\max 2}}{F_{\text {block } 2}}-M^{2} \frac{d_{\max 1}}{F_{\text {block } 1}}\right)^{2} \\
& \text { such that } \\
& \sigma_{\text {axial }}-\sigma_{\text {buckling }} \leq 0 ; \quad V-V_{\text {allow }} \leq 0 \\
& h_{\min } \leq h_{i} \leq h_{\max } ; \quad X_{\min } \leq X_{j} \leq X_{\max } \\
& Y_{\min } \leq Y_{k} \leq Y_{\max }
\end{aligned}
$$

\footnotetext{
${ }^{1}$ Corresponding author: 1415 Engineering Drive, Madison, WI 53706-1691; Tel: (608) 262-2233, Fax: 262-1267, E-mail: yogesh@engr.wisc.edu
} 
The required magnification factor, $M$, is enforced by penalizing the objective function by the penalty factor, $P$. Buckling is enforced by limiting the maximum axial stress to $\sigma_{\text {buckling }}$ based on Euler's buckling criteria, modified for end conditions (during dimensional synthesis). The total volume constraint is enforced by $V_{\text {allow. }}$. Design variables include element width and $\mathrm{X}$ and $\mathrm{Y}$ node position, denoted by $h_{i}, X_{j}$, and $Y_{k}$ respectively. These are given local upper and lower bounds which serve to limit the search space and also to enforce fabrication limitations (during dimensional synthesis).

\section{Topology Synthesis}

To establish the optimal topology of a mechanism, the design domain is discretized using a network of truss elements, defined as a ground structure. The topology optimization process seeks to resize each element thickness until the performance of the structure is maximized. Upon completion, elements which converge to the lower bound are removed yielding the final mechanism topology. The topology synthesis approach described here utilizes a modular ground structure where nodes are allowed to change location within 'wandering limits'. Research has shown that this technique produces good topology designs that accurately satisfy magnification requirements [9].

Figure 2 illustrates the example compliant mechanism design problem. Given a positive actuator input force, the output is designed to move in the negative vertical direction. Taking advantage of symmetry allows only half of the topology to be designed, which lowers computational requirements. For the inverter example, the modulus was set to 1000 , the width was set to 1 , the total volume constraint was set to 2 , and the lower element bound was set at $1 \mathrm{E}-4$. To achieve the magnification factor of 20 , the penalty multiplier was set to 1 . Figure 3 shows the floatingnode ground structure, discretized using 25 nodes and 72 elements; 23 nodes were activated and given wandering ranges resulting in 107 design variables (wandering ranges indicated by gray areas). Figure 4 indicates the optimal topology of the compliant mechanism amplifier with elements converging to the lower bound removed.

\section{Dimensional Synthesis}

To proceed with dimensional synthesis, each segment of the topology is re-meshed using arrays of beam elements; the number of elements along an array is directly controlled by the designer. Exact specifications for the material modulus, out-of-plane thickness, input actuator characteristics, and desired magnification are input into the optimization routine. The designer may choose to activate end nodes of topology segments, allowing for geometric variation. Intermediate nodes along the length of an activated segment are adjusted by maintaining a collinear equidistant relationship.

Figure 5 shows the inverter example from the topology synthesis remeshed for the secondary dimensional synthesis stage. The mechanism is to be fabricated using electroplated nickel as the structural material. Constraints within the fabrication sequence limit the minimum thickness and width of the structure to about $15 \mu \mathrm{m}$. The mechanism is designed to be driven using a nickel bent-beam actuator ( $H$ $=W=15 \mu \mathrm{m}, L=1000 \mu \mathrm{m}$ ) operating at a temperature difference of $200{ }^{\circ} \mathrm{C}$ with respect to the substrate $\left(F_{\text {block }}=\right.$ $\left.25000 \mu \mathrm{N}, d_{\max }=6.8 \mu \mathrm{m}\right)$. The thermal expansion coefficient of the metal is approximately $13.5 \mathrm{ppm} / \mathrm{K}[10]$. Figure 6 shows the optimized structure $(M=20, P=1$, $\left.V_{\text {allow }}=1 \times 10^{6} \mu \mathrm{m}^{3}\right)$. This particular design offers a blocking force of $470 \mu \mathrm{N}$ (assuming a Young's modulus of $100 \mathrm{GPa}$ ) and a free displacement of $51 \mu \mathrm{m}$. Because of the minimum width limitation, substantial energy is spent deforming the compliant transmission (transmission efficiency is 14.1 percent). Larger blocking force and free displacement can be achieved by fabricating more flexible structures with thinner beam widths (within buckling limilations).

\section{EXPERIMENTAL RESULTS}

Thermal actuators with microtransmissions were fabricated on silicon wafers insulated with thermal oxide and LPCVD nitride. A $2.2 \mu \mathrm{m}$ thick sputtered Ti sacrificial layer was patterned and covered with a $\mathrm{Cr} / \mathrm{Ni}$ seed layer. The devices were electroplated into a photoresist mold from a nickel sulphamate solution. At $55^{\circ} \mathrm{C}$ temperature, the plating current was adjusted to provide a structural layer thickness of $16 \mu \mathrm{m}$ in $120 \mathrm{~min}$. Devices with other thicknesses were also electroplated at the same rate. The photoresist mold was subsequently stripped and the sacrificial material etched away. Some samples were then covered with a thin layer of gold by electroless plating to provide an inert coating [10]. Following this, a coating of sclf-asscmbled monolayers was applicd. Optical micrographs of a fabricated structures are shown in Fig. 7.

The dimensional parameters of designs that were fabricated are defined in Fig. 8 and listed in Table I. All fabricated devices were tested electrically. A sample set of measurements is plotted in Fig. 9, showing the displacement at the input and output of the microtransmission. The measurements were taken by a calibrated visual method, with an uncertainty of $<0.5 \mu \mathrm{m}$. The measured displacement amplification, $A$, for this device was 3.1, which is very close to theoretical prediction of 3.2. Electrothermal tests of a device that was $16 \mu \mathrm{m}$ thick but similar to design $2 \mathrm{a}$ in all other respects showed an output displacement of $9.7 \mu \mathrm{m}$ at a drive voltage of $0.6 \mathrm{~V}$. Due to the high $A$ offered by devices $2 \mathrm{a}$ and 3 , the displacement at the input to the microtransmission was not measurable at the peak operating temperatures. In order to verify theoretically predicted $A$ of these devices, the microtransmissions were mechanically actuated with a probe tip. The results matched expectations within $10 \%$. Sample measurements for device 3 are shown in Fig. 10.

Note that the output blocking forces are linearly related to the Young's modulus of the structural material. When electroplated metals are used, there is significant variability in this parameter [10]. Values ranging from $35 \mathrm{Gpa}$ to 150 $\mathrm{GPa}$ hav been reported for electroplated nickel, whereas the Young's modulus of bulk nickel is $208 \mathrm{GPa}$.

\section{CONCLUSIONS}

Microtransmissions have been used to tailor the forcedisplacement relationships of electro-thermal actuators. The microtransmissions are flexural elements that provide backlash-free operation and rectilinear input and output displacements that are suitable for use with bent-beam actuators and convenient for numerous applications. Topology synthesis and dimensional synthesis are demonstrated to effectively automate the design of compliant mechanisms obtaining extreme magnification 
values. The two-stage process produces detailed designs given actuator and material input parameters while considering magnification, buckling, and minimum width constraints.

The resulting electro-thermal actuators with integrated microtransmissions were fabricated by electroplating nickel on silicon substrates. Electrical and mechanical tests confirmed the model predicitons, with the measured multiplication factor matching the theoretical value to within $10 \%$. Displacement amplification factors of 3-14 have been demonstrated. However, these are constrained by the modest fabrication limits, and higher performance can be achieved.

\section{REFERENCES}

[1] L. Que, J.-S. Park, Y.B. Gianchandani, "Bent-Beam ElectroThermal Actuators for High Force Applications," IEEE Intl. Conf. on Micro Electro Mechanical Systems (MEMS99), Orlando, Florida, Jan. 1999

[2] J.-S. Park, L.L Chu, E. Siwapornsathain, A.D. Oliver, Y.B. Gianchandani, "Long Throw and Rotary Output ElectroThermal Actuators Based on Bent-Beam Suspensions," IEEE Intl. Conf. on Micro Electro Mechanical Systems (MEMSOO), Miyazaki, Japan, Jan. 2000

[3] S. Kola, J. Hetrick, Z. Li, L. Saggere, "Tailoring Unconventional actuators using compliant transmissions: Design Methods and Applications," IEEE/ASME Transactions on Mechatronics, 4(4), 1999, pp. 396-408

[4] S. Nishiwaki, M. Frecker, M. Seungjae, N. Kikuchi, "Topology Optimization of Compliant Mechanisms Using the Homogenization Method,", Intl. Journal for Numerical Methods in Engineering, 42(3), 1998, pp. 535-559

[5] O. Sigmund, "Tailoring Materials with Prescribed Elastic Properties," Mechanics of Materials, v. 20, 1995, pp. 351-368

[6] G. Ananthasuresh, S. Kota, N. Kikuchi, "Strategies for Systematic Synthesis of Compliant MEMS," Dynamic Systems and Control, DSC-Vol. 55-2, 1994 ASME Winter Annual Meeting, Chicago, IL, Nov. 1994, pp. 677-686

[7] T. Moulton, G. Ananthasuresh, "Micromechanical Devices with Embedded Electro-Thermal-Compliant Actuation," MEMS-Vol. 1, ASME International Mechanical Engineering Conference and Exposition, MEMS, Nov. 1999, Nashville, Tenessee, pp. 553-560

[8] J. Jonsmann, O. Sigmund, S. Bouwstra, "Compliant Thermal Microactuators," Sensors and Actuators (A), Vol. 76, 1999, pp. $463-469$

[9] J. Hetrick, A Unified Approach for Topological and Dimensional Synthesis of Compliant Mechanisms, Ph.D. Thesis, University of Michigan, Ann Arbor, Michigan, 1999

[10] L. Chu, L. Que, Y. Gianchandani, "Temperature Coefficients of Material Properties Using Differential Capacitive Strain Sensors," Proc., Solid-State Sensors and Actuators Workshop, Hilton Head, S.C., June 2000

Table I: Summary of simulated and measured results. Dimensions are defined in Fig. 8. $H$ is device thickness; $d_{\max }$ and $F_{\text {block }}$ are calculated at the output, assuming a $200^{\circ} \mathrm{C}$ temp. difference with respect to the substrate, and the Young's modulus is $100 \mathrm{GPa}$.

\begin{tabular}{|c|c|c|c|c|c|c|c|c|}
\hline $\begin{array}{c}\text { Dev } \\
\#\end{array}$ & $\begin{array}{c}\mathrm{L} 0 \\
\mu \mathrm{m}\end{array}$ & $\begin{array}{c}\mathrm{L} 1 \\
\mu \mathrm{m}\end{array}$ & $\begin{array}{c}\mathrm{W} \\
\mu \mathrm{m}\end{array}$ & $\begin{array}{c}\mathrm{H} \\
\mu \mathrm{m}\end{array}$ & $\begin{array}{c}\mathrm{d}_{\max } \\
\mu \mathrm{m}\end{array}$ & $\begin{array}{c}\mathrm{F}_{\text {block }} \\
\mu \mathrm{N}\end{array}$ & $\mathrm{A}_{\text {calc }}$ & $\mathrm{A}_{\text {meas }}$ \\
\hline 1 & 720 & 1500 & 70 & 35 & & & 3.2 & 3.1 \\
\hline $2 \mathrm{a}$ & 1000 & 750 & 10.8 & 9.7 & 31.5 & 244 & 11.1 & 10.6 \\
\hline 3 & 1000 & 1000 & 10.8 & 16.0 & 134 & 394 & 14.1 & 12.9 \\
\hline
\end{tabular}

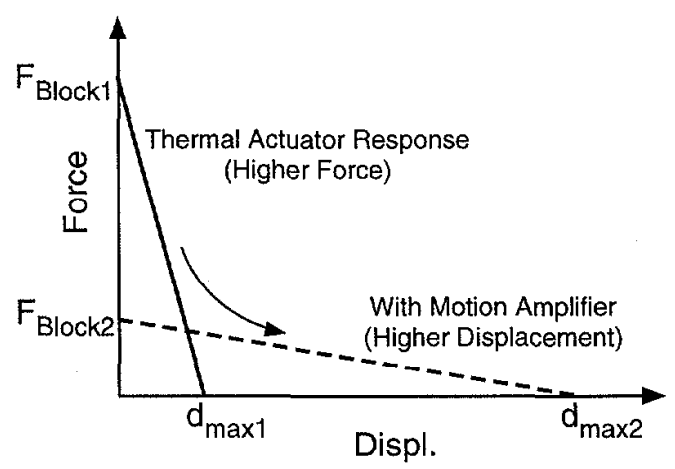

Fig. 1: A compliant micro-transmission provides rectilinear motion and allows the force-displacement trade-off to be tailored for an application.

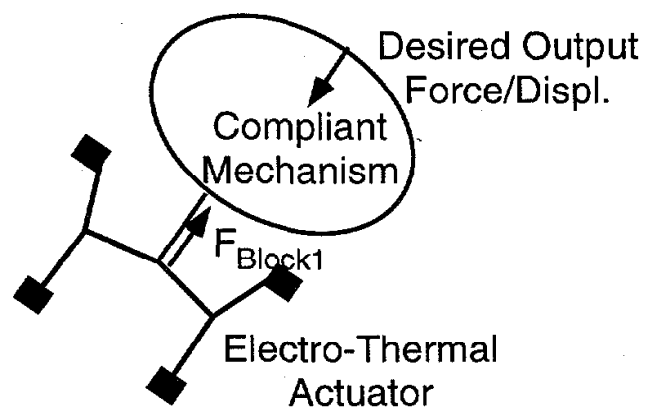

Fig. 2: The topology design problem for a compliant mechanism rectilinear amplifier.

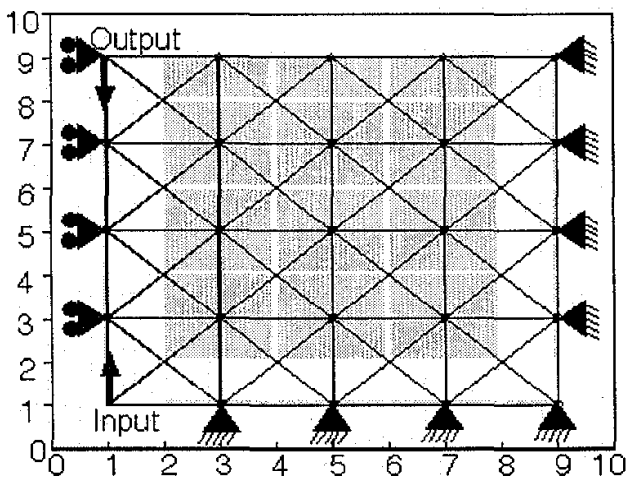

Fig. 3: Half of the design domain is meshed using truss elements (gray areas denote wandering regions).

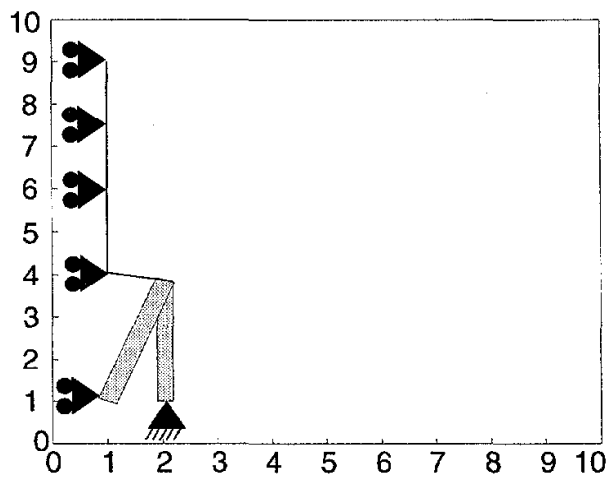

Fig. 4: The optimized mechanism topology. 


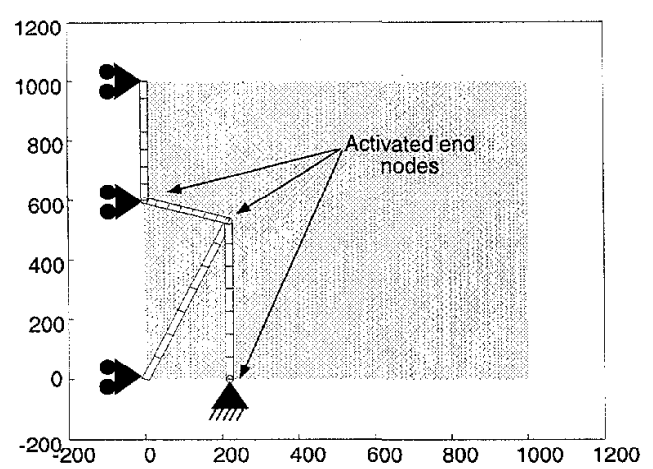

Fig. 5: The dimensional synthesis problem with the structure modeled using beam elements (gray areas denote end node wandering ranges).

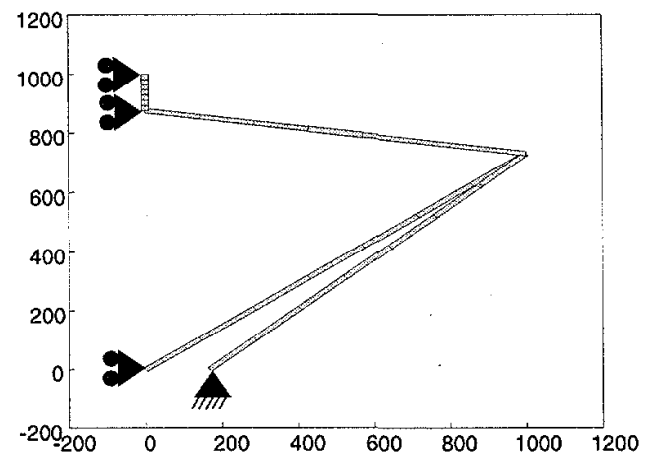

Fig. 6: Half of the finalized compliant mechanism.
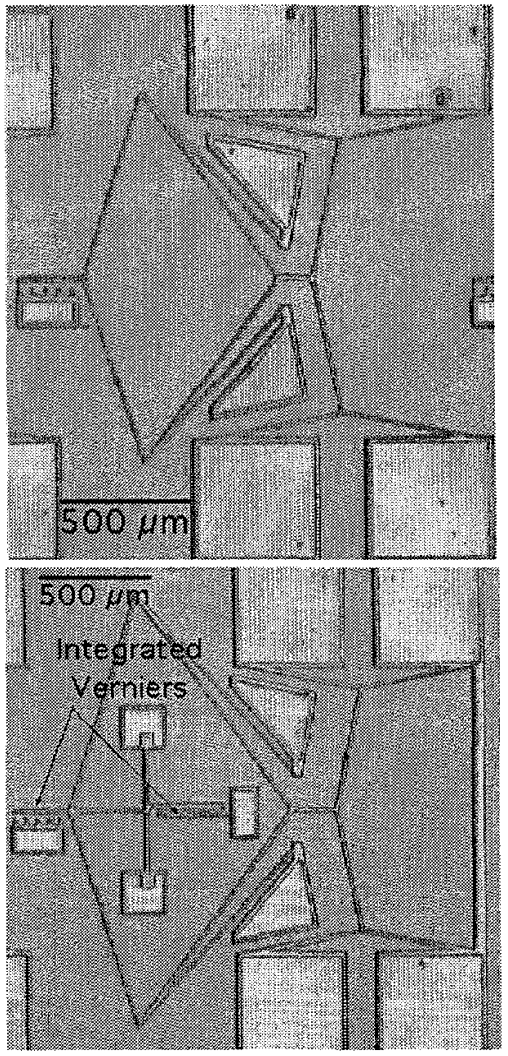

Fig. 7: Optical micrographs of cascaded bent-beam actuators with attached microtransmissions. The devices were fabricated from $\mathrm{Ni}$ electroplated on Si substrates.

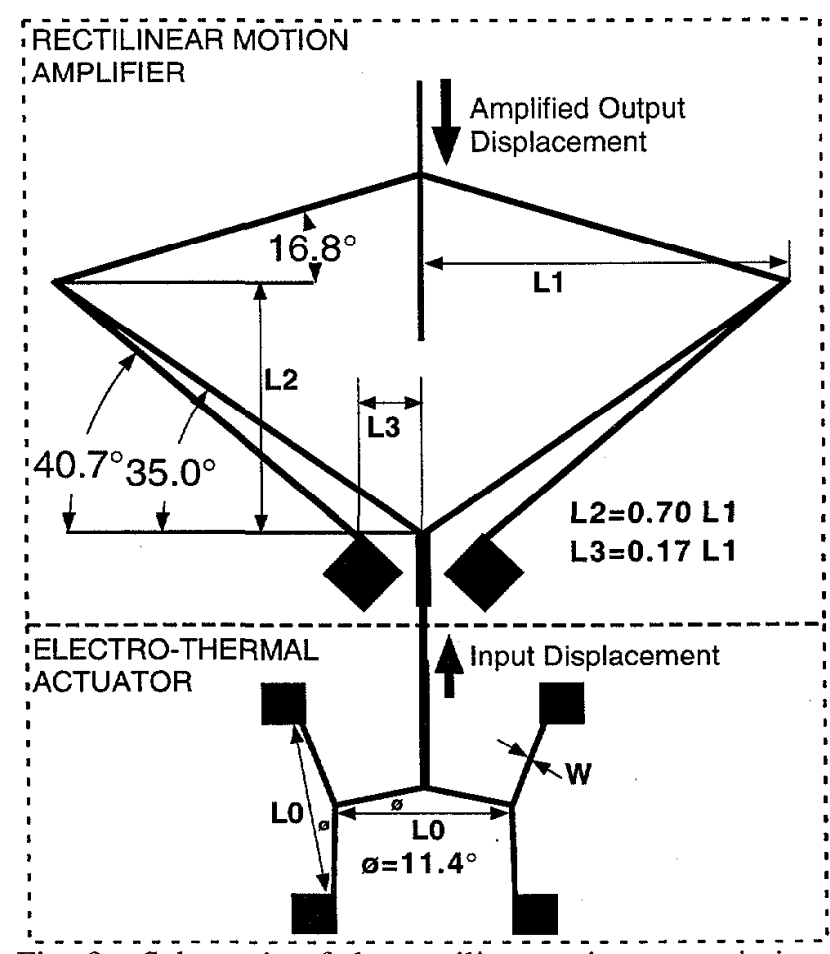

Fig. 8: Schematic of the rectilinear microtransmission and cascaded actuator showing the dimensional variables.

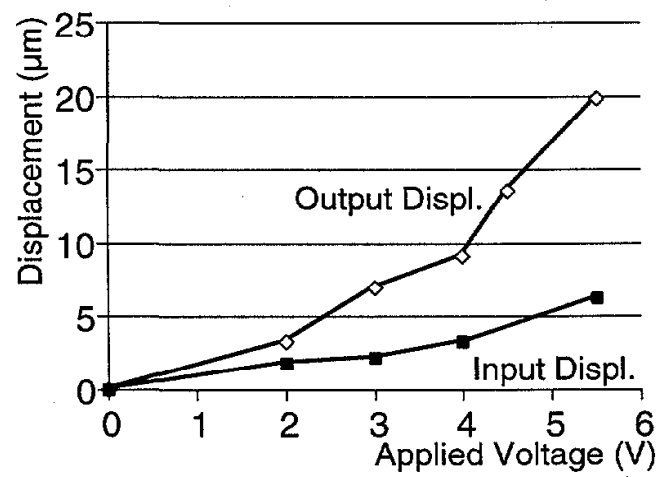

Fig. 9: Electro-thermally generated displacements measured at the input and output of the transmission of device 1 , with dimensional parameters as in Table I.

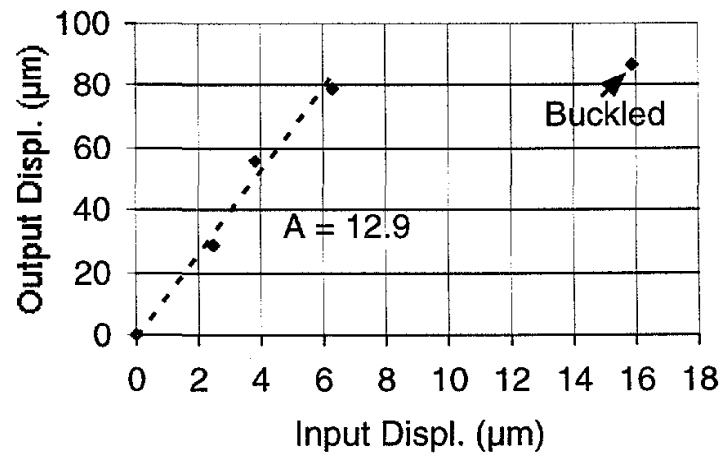

Fig. 10: Measured ratio of input to output displacement for the microtransmission of device 3 (Table I). This measurement was taken mechanically because of operating temperature limitations. 